\title{
Creation of an interdisciplinary Burn
Center log book
}

cHuV

Dan Carel $^{1}$, Mette M. Berger ${ }^{1}$, Olivier Pantet ${ }^{1}$, Stéphanie Terrier ${ }^{1}$, Aline Schmid ${ }^{1}$ ${ }^{1}$ Service of Adult Intensive Care Medicine and Burns, Lausanne University Hospital (CHUV), Rue du Bugnon 46, 1011 Lausanne, Switzerland.

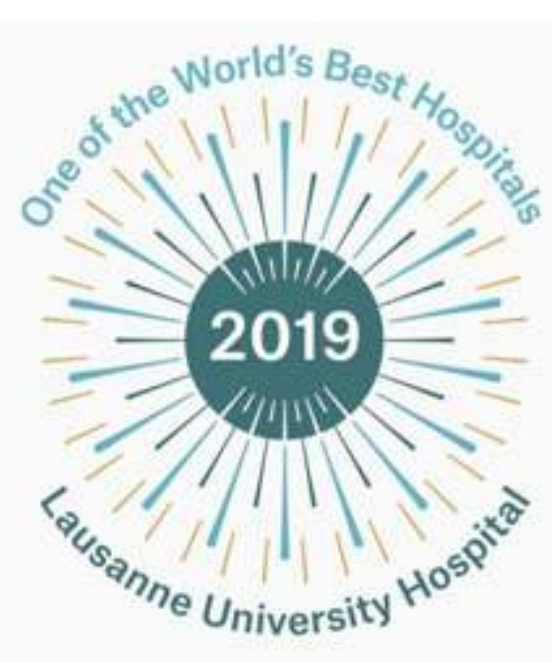

\section{Background and objectives}

Many documents, procedures or protocols describe the practices at the burn centre in Lausanne (Switzerland). The need to collect them and hold them in the pocket in an easily accessible way was a caregivers' need.

The main objective was to generate a handy pocket document integrating all disciplines and treatments from admission to the burn centre until discharge and rehabilitation.

\section{Methods}

To address the very different needs of physicians, surgeons and PAM, interdisciplinary meetings were organised. Existing protocols and procedures were collected, and updated. Protocols had been generated for the EBA verification in 2015, but existed as multiple separate documents. Several meetings were organized between 2016 and 2018 to promote the integration of recent literature references (PubMed, Cinhal). Agreement process was by consensus after multiple circulation of documents. They were grouped by phase of treatment and by organ care, more than by specialty which was a novel approach. The final document was again circulated, and sent for printing after validation. An electronic version was kept as
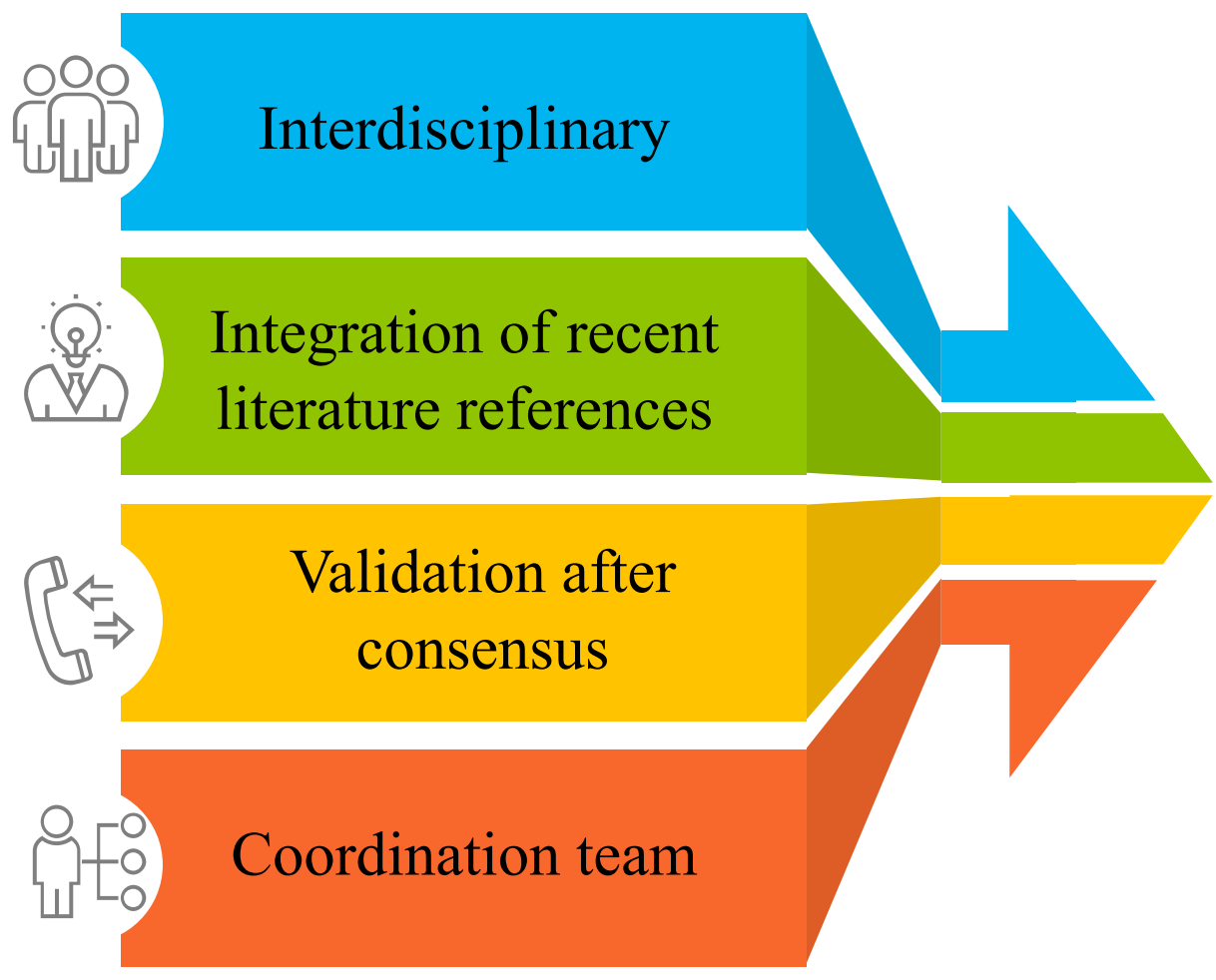

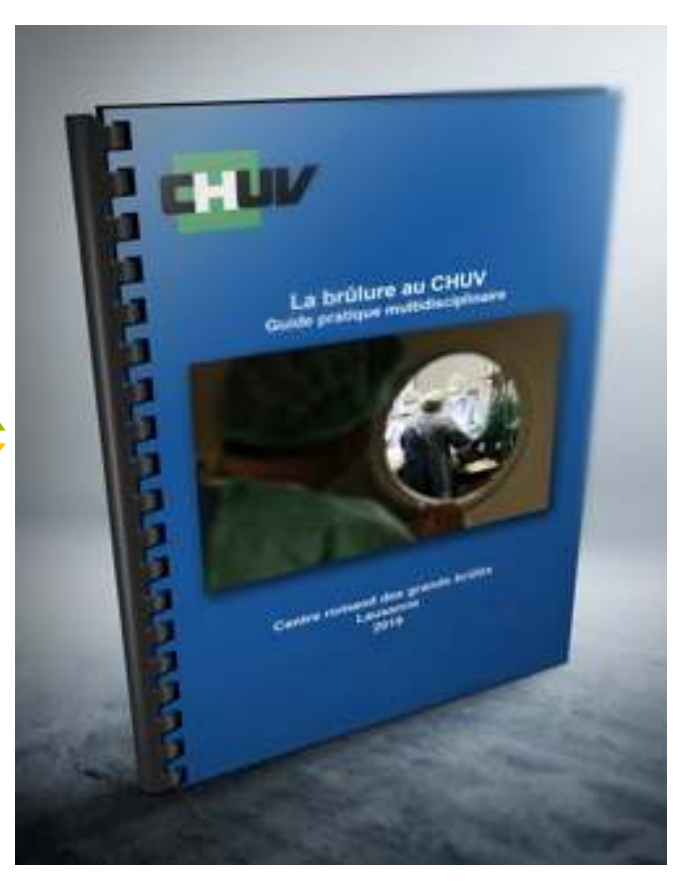

\section{Results}

The final document includes 162 pages, multiple figures and photographs, and 53 references. Format is A6, enabling pocket placing. Chapters are : admission, respiratory care, cardiovascular, neurologic, environment, renal, digestive, surgical and topical treatment. The publication generated an intense team enthusiasm. The book enabled a general view of burn care that generated several constructive comments for the future developments.

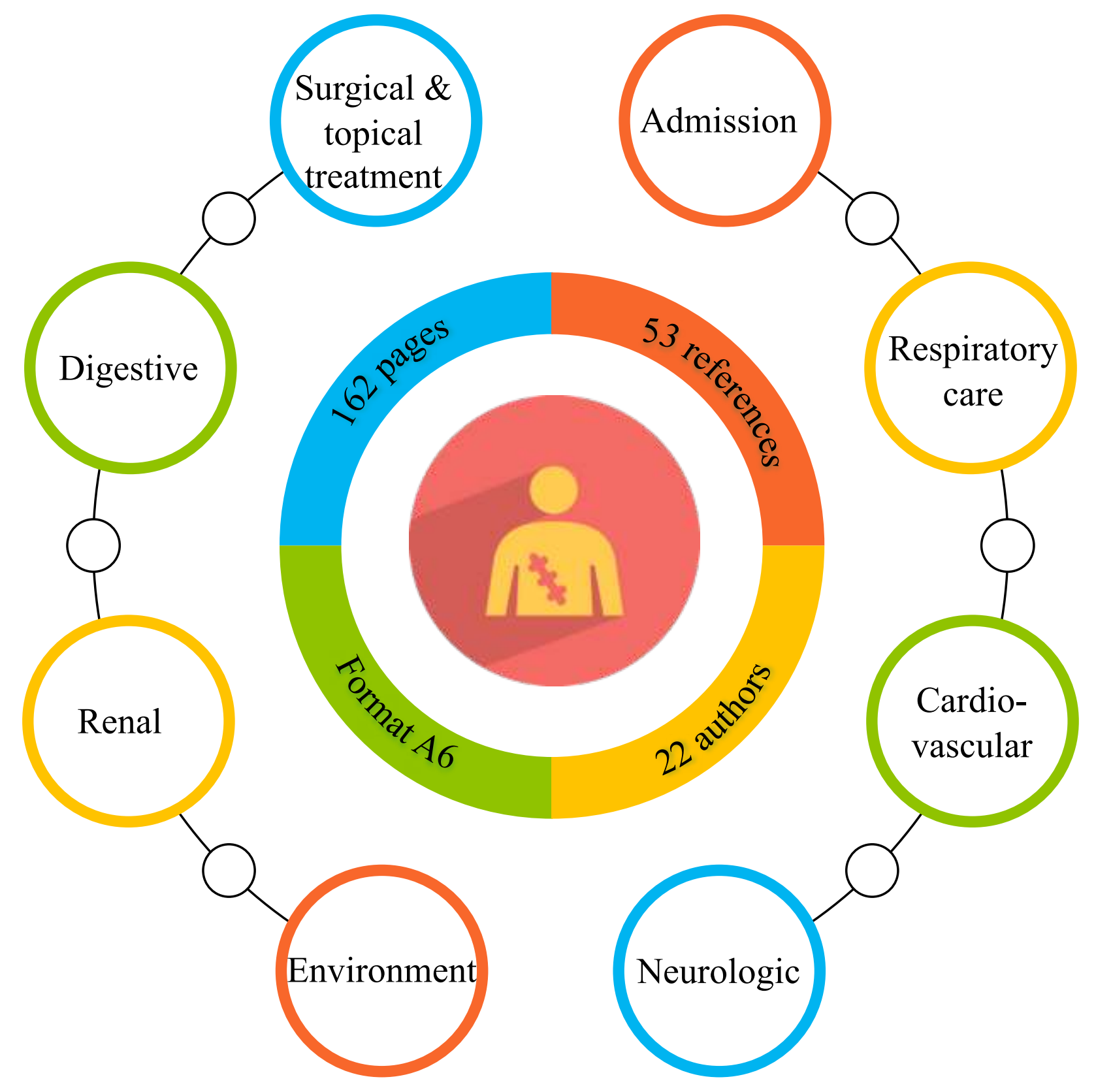

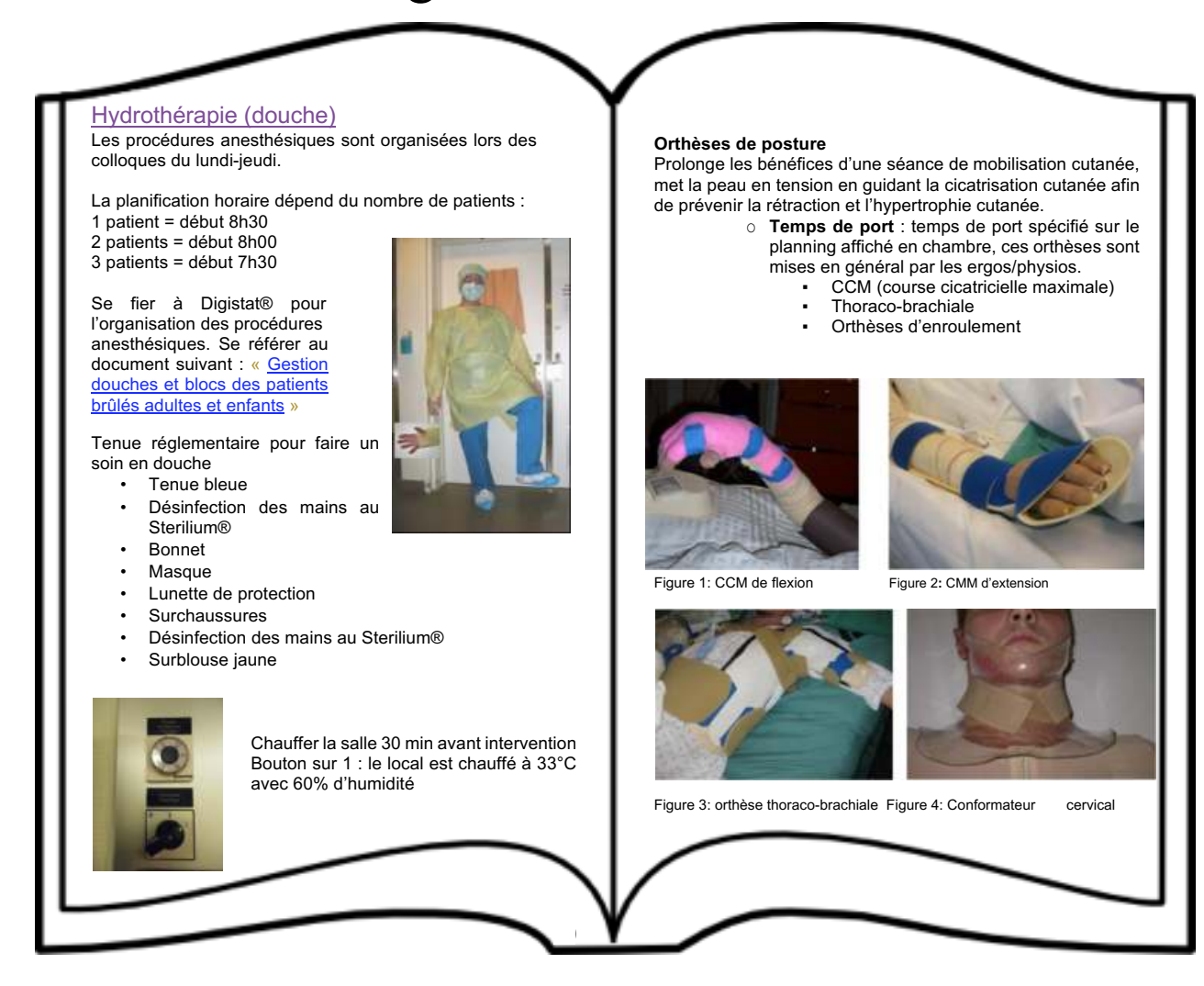

Conclusion

The book enabled a general view of our practices that gave recognition of every involved specialist. While the creation required heavy work, the result was rewarding and enforced the team feeling. The book is meaningful to the team as to the patient needs. 